\title{
An Observer-Based Friction Compensation Technique for Positioning Control of a Pneumatic Servo System*
}

\author{
Takahiro KOSAKI** and Manabu SANO** \\ ** Department of Systems Engineering, Hiroshima City University \\ 3-4-1 Ozuka-higashi, Asaminami-ku, Hiroshima 731-3194, Japan
}

\begin{abstract}
This paper presents a technique to cope with undesirable frictional effects in positioning control of a pneumatic actuator. This technique is based on an observer for estimating the dynamically varying friction force on-line. The observer is derived from an available nonlinear observer for Coulomb friction by modifying it to fit the pneumatic actuator and to enhance its estimation ability. Our pneumatic servo system for positioning consists of an inner pressure control loop, an outer position control loop, the friction observer, and a velocity observer. In this system, the friction force is compensated by adjusting a desired pressure value sent from the outer loop to the inner loop according to the friction observer output. Experimental comparisons with a conventional control system using friction compensation by means of accelerometer information feedback were carried out and show that our system works with almost the same high positioning accuracy as the conventional system, despite having neither an accelerometer nor a velocity sensor, and is more advantageous from the perspective of cost performance.
\end{abstract}

Key words : Oil and Air Hydraulics, Pneumatic Actuator, Servo Mechanism, Positioning, Observer, Friction Compensation

\section{Introduction}

Pneumatic actuators are more appropriate for medical and wearable purposes involving direct contact and interaction with humans than electric and hydraulic actuators because they have a high power-to-weight ratio and high mechanical compliance due to the compressibility of air and they are applicable in clean conditions. In order to meet these purposes sufficiently, functional enhancements such as position control and force control are often required but are difficult due to the presence of various nonlinear factors. Above all, friction forces occur in a nonlinear and uncertain manner in the seals for avoiding air leakage and may cause unfavorable stick-slip oscillation at extremely low operating speeds. Due to such behavior, a classical linear controller can only achieve a limited performance. In recent years, advanced control strategies such as robust control have attracted much attention, and many papers have been presented so far $^{(1)-(4)}$. On the other hand, more efforts to investigate friction compensation approaches are also required ${ }^{(5)-(7)}$.

Focusing on gain-scheduled $H_{\infty}$ control that is capable of stabilizing a closed-loop system despite the inclusion of time-varying parameters, we have proposed a technique for compensating for friction in a pneumatic actuator system by dealing with the dynamic friction coefficient as a time-varying parameter ${ }^{(8)}$. This technique requires mathematical modeling of the actual friction characteristics in advance, and the control performance depends to some extent on the accuracy of the model. For general mechanical systems, Friedland and Park ${ }^{(9)}$ have developed a nonlinear observer for estimating the friction force and a friction compensation method that utilizes this observer. Their method does not require modeling the actual 


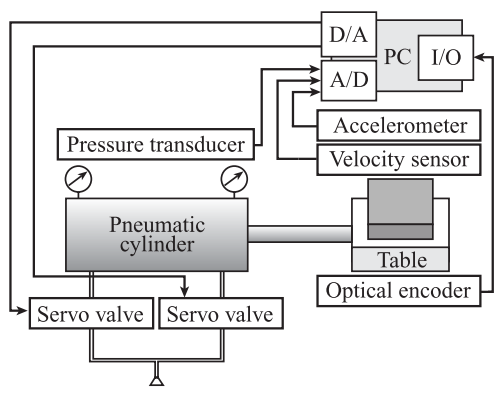

Fig. 1 Arrangement of experimental apparatus

friction and is able to compensate for even unexpected dynamic fluctuations in the friction. The effectiveness of the method was exhibited by applying it to a journal bearing driven by an electric motor ${ }^{(10)}$ and to a hydraulic manipulator ${ }^{(11)}$.

In the present study, the friction observer proposed by Friedland and Park in Ref. (9) was modified and applied to friction compensation in the positioning control system of a pneumatic actuator. In the modification phase, a component describing the effect of pressure inside the actuator chambers on the friction at the seal is incorporated into the observer. The estimation accuracy of the resulting observer was examined experimentally. In the application phase, we designed a pneumatic servo system consisting of an inner pressure proportionalderivative (PD) control loop ${ }^{(12)}$, an outer position PD control loop, the friction observer, and a velocity observer. The friction compensation is executed by adjusting a desired pressure value sent from the outer loop to the inner loop according to the friction observer output. Its performance was evaluated based on the positioning errors in the experiments. Additionally, experimental comparisons with a pneumatic servo system equipped with an accelerometer and a velocity sensor were conducted to confirm that our system without these costly sensors is more advantageous from the perspective of cost performance, as reducing the number of sensors increases the potential to make pneumatic actuators competitive with other power source actuators, at least from a cost perspective.

\section{Control system design}

\subsection{Experimental system}

Figure 1 illustrates our experimental apparatus, which is principally composed of a standard pneumatic cylinder, two proportional control valves, and a PC for implementing a control algorithm. A pneumatic cylinder with a single piston rod has a piston diameter of $50 \mathrm{~mm}$ and a stroke length of $400 \mathrm{~mm}$. It is equipped with squeeze-type seals on the sliding surfaces. A table onto which weights are loaded is connected to the piston rod. The table is sustained by linear guides using ball bearings with a friction coefficient of approximately 0.001 . The position of the piston is detected by an optical encoder with a resolution of $5 \mu \mathrm{m}$, and its signal is converted to a digital code in a counter. To determine the velocity of the piston, a sensor is used that produces a voltage proportional to the velocity while a magnet is moving through the coils. The cylinder chamber pressure is measured by two pressure transducers. The proposed controller is executed on a PC with digital-I/O, A/D, and D/A interfaces. Control signals, which are computed on the PC from the outputs of the sensors, are sent to the control valves. In addition, the acceleration information required in the calculation of an actual friction force is acquired by an accelerometer mounted on the piston.

\subsection{Pressure controller}

Figure 2 shows the basic structure of our control system, which is composed of an inner pressure feedback loop and an outer position feedback loop.

The purpose of the controlling pressure is to reduce the time delay in a chamber pressure change to a control command signal. Under the assumption of an adiabatic change of a perfect 


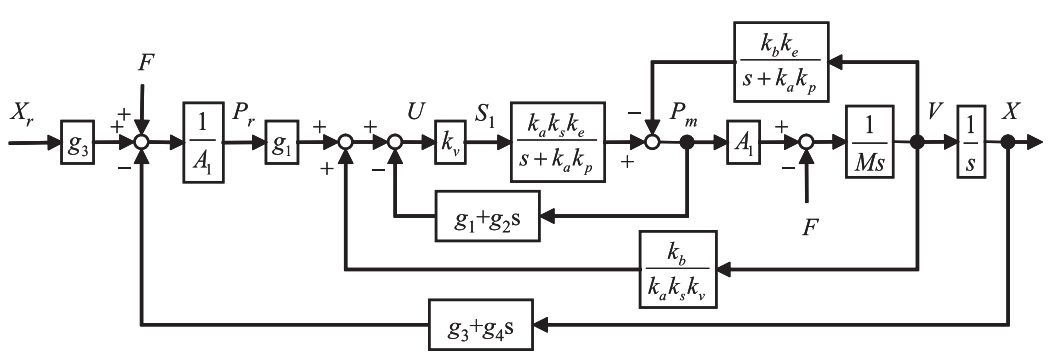

Fig. 2 Structure of a pneumatic servo system

gas, the equation describing the pressure inside the chamber on either side of the piston in a pneumatic cylinder is as follows ${ }^{(12)}$ :

$$
\begin{aligned}
& \dot{p}_{1}=\frac{\kappa R T}{V_{1}} G_{1}\left(s_{1}, p_{1}\right)-\frac{\kappa A_{1} p_{1}}{V_{1}} v \\
& \dot{p}_{2}=\frac{\kappa R T}{V_{2}} G_{2}\left(s_{2}, p_{2}\right)+\frac{\kappa A_{2} p_{2}}{V_{2}} v
\end{aligned}
$$

where $p$ is the pressure inside the chamber, $G$ is the mass flow rate through the control valve, $s$ is the effective sectional area of the control valve, $V$ is the volume of the chamber, $T$ is the temperature, $R$ is the gas constant, $A$ is the piston sectional area, $\kappa$ is the specific heat ratio, and $v$ is the piston velocity. The subscripts 1 and 2 denote the left and right chambers in Fig. 1 , respectively.

We assume the center of the piston's range of movement to be an equilibrium point. Linearizing Eqs. (1) and (2) at this point, we get

$$
\Delta \dot{p}_{m}=k_{a}\left(\Delta G_{1}-k_{d} \Delta G_{2}\right)-k_{e} k_{b} \Delta v
$$

where $p_{m}=p_{1}-k_{d} p_{2}, k_{d}=A_{2} / A_{1}, k_{a}=\left(\kappa R T_{0}\right) / V_{0}, k_{b}=\left(\kappa A_{1} p_{0}\right) / V_{0}$, and $k_{e}=1+k_{d}$. The subscript 0 indicates a value at the equilibrium point, and $\Delta$ denotes infinitesimally small changes from that point.

Defining $k_{s i}=\left(\partial G_{i} / \partial s_{i}\right)_{0}$ and $k_{p i}=-\left(\partial G_{i} / \partial p_{i}\right)_{0}$, the linearized equation of the mass flow rate is written as

$$
\Delta G_{i}=k_{s i} \Delta s_{i}-k_{p i} \Delta p_{i}
$$

In the equilibrium point, we suppose that $k_{s 1}=k_{s 2}=k_{s}, k_{p 1}=k_{p 2}=k_{p}$, and $\Delta s_{1}=-\Delta s_{2}=$ $\Delta s$. After substituting Eq. (4) into Eq. (3) and applying the Laplace transformation, we finally obtain

$$
P_{m}(s)=\frac{k_{e}\left\{k_{a} k_{s} S(s)-k_{b} V(s)\right\}}{s+k_{a} k_{p}}
$$

where a capital letter indicates a Laplace-transformed variable.

Approximating the dynamics of a control valve as $S(s)=k_{v} U(s)$, we apply the following PD-feedback control law to Eq. (5):

$$
U(s)=g_{1}\left\{P_{r}(s)-P_{m}(s)\right\}-g_{2} s P_{m}(s)+U_{c}(s)
$$

where $U(s)$ is the operating voltage of the control valve, and $P_{r}(s)$ is the desired pressure. $U_{c}(s)$ is added to Eq. (6) to cancel the velocity term in Eq. (5), which is given by $U_{c}(s)=$ $\left\{k_{b} V(s)\right\} /\left(k_{a} k_{s} k_{v}\right)$. The tracking performance for the desired pressure can be adjusted by the gains, $g_{1}$ and $g_{2}$. 


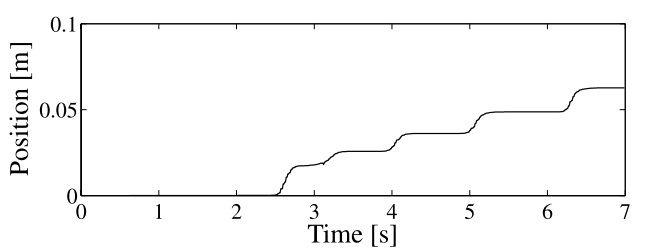

(a) Measured position

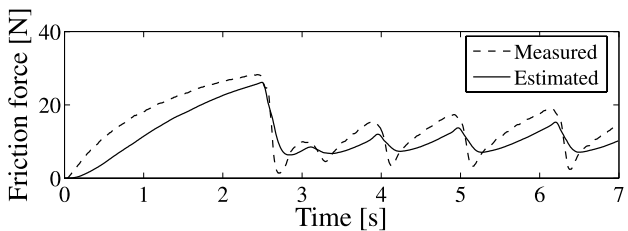

(b) Measured and estimated friction

Fig. 3 Estimation of friction using the original observer

\subsection{Position controller}

The equation of motion of the moving part in the apparatus illustrated in Fig. 1 is represented by

$$
M a=A_{1}\left(p_{1}-p_{a}\right)-A_{2}\left(p_{2}-p_{a}\right)-f
$$

where $a$ is the piston acceleration, $M$ is the total mass of the moving part, $f$ is the friction force, and $p_{a}$ is the atmospheric pressure. Applying the Laplace transformation to the linearized equation of Eq. (7), we get

$$
M s^{2} X(s)=A_{1} P_{m}(s)-F(s)
$$

where $X(s)(=\mathcal{L}[x])$ is the piston position. If the response of the pressure control using Eq. (6) is much faster than the piston motion, we can assume $P_{m}(s)=P_{r}(s)$ in Eq. (8). In this case, positioning control can be performed by setting the desired pressure with the following PD-feedback law ${ }^{(9),(13)}$ :

$$
P_{r}(s)=\left\{g_{3} E(s)-g_{4} s X(s)+F(s)\right\} / A_{1}
$$

where $E(s)=X_{r}(s)-X(s)$, and $X_{r}(s)$ is the desired position. Hence, assuming that the pressure control system ideally acts and its dynamics can be neglected, the position control system can be expressed in the following pressure-independent form:

$$
X(s)=\frac{g_{3} / M}{s^{2}+\left(g_{4} / M\right) s+\left(g_{3} / M\right)} X_{r}(s)
$$

where $g_{3}$ and $g_{4}$ are the gains that allow us to tune the positioning performance.

The position control law consists of Eqs. (6) and (9). Acquisition of the value of the friction force included in Eq. (9) is provided in the next section.

\section{Friction estimation}

\subsection{Friction observer}

In the proposed controller, highly precise acquisition of the nonlinear friction force $F(s)$ in Eq. (9) contributes to enhancement of the positioning performance. One way to achieve this is the on-line estimation of the friction force using an observer. Friedland and Park ${ }^{(9)}$ have proposed the following observer to estimate the friction force in mechanical systems:

$$
\begin{aligned}
& \hat{f}=M\left\{z-g_{a}|v|^{\mu}\right\} \operatorname{sgn}(v) \\
& \dot{z}=g_{a} \mu|v|^{\mu-1} \hat{a} \operatorname{sgn}(v)
\end{aligned}
$$

where $\hat{f}$ is an estimate of the friction force, $z$ is the state variable, $\operatorname{sgn}(\cdot)$ is a signum function, $\hat{a}$ is defined as $\hat{a}=\left(f_{a}-\hat{f}\right) / M$, where $f_{a}$ is a driving force, and $g_{a}$ and $\mu$ are the design 


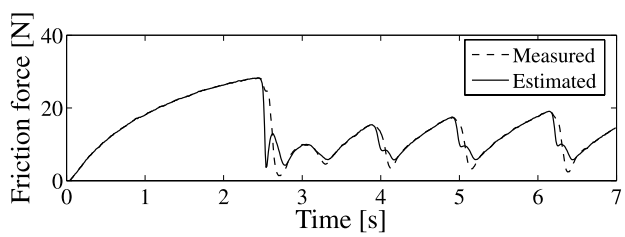

Fig. 4 Estimation of friction using the observer with a static friction component

parameters to determine the estimation performance. In this observer, it is guaranteed that the estimation error converges to zero if $g_{a}>0, \mu>0$, and $v \neq 0$, as long as we regard the friction force as a constant, i.e., $\dot{f}=0$.

In the practical use of this observer, it is necessary to identify the design parameters in Eqs. (11) and (12) from experimental data for different operating conditions. In this study, we used the apparatus shown in Fig. 1 to acquire the data set, namely a pneumatic cylinder was driven by the application of various constant voltages to the control valves and the open-loop responses were recorded. Direct measurement of the actual friction force is difficult, but it can be calculated by the following equation based on Eq. (7) from the data:

$$
f=A_{1} p_{1}^{*}-A_{2} p_{2}^{*}-M a^{*}
$$

The superscript $*$ in Eq. (13) indicates a value measured directly by each sensor. On the other hand, the estimated value of the friction force $\hat{f}$ can be obtained by inputting the data into Eqs. (11) and (12) and by solving them numerically. The design parameters, $g_{a}$ and $\mu$, included in Eqs. (11) and (12) are selected so that $\hat{f}$ can approach $f$ according to the following instructions $^{(11)}$ :

- Although the friction observer is nonlinear, its pole is approximately given by $\epsilon=$ $-g_{a} \mu \overline{|v|^{\mu-1}}$ by analogy with a linear time-invariant observer. $\overline{(\cdot)}$ denotes an average value. Based on this, $\mu$ is selected in $1<\mu<2$ to achieve fast estimation when the average velocity is extremely low.

- The value of the pole $\epsilon$ is determined such that $\epsilon$ is at least four times larger than that of a control system.

In some preliminary trials in which we off-line calculated the estimated value by using the friction observer from the experimental data and compare it with the actual value, approaching the value of $\mu$ to 2 raised the estimation accuracy at high velocities, while the accuracy deteriorated at low velocities because higher piston velocity results in a larger observer pole. When $\mu$ approached 1 , the results showed the reverse tendency. With regard to $g_{a}$, after we roughly determined $g_{3}$ and $g_{4}$ by simulations, we got $g_{a} \geq 40$ based on the pole of Eq. (10) and the above instructions. As a result, by considering not only the estimation accuracy but also the sensitivity to noise, we set the design parameters; i.e., $\mu=1.5$ and $g_{a}=50 \mathrm{~m}^{-1 / 2} \mathrm{~s}^{-1 / 2}$ were chosen.

The results of the off-line estimation tests using the friction observer are shown in Fig. 3. These results were obtained when stick-slip oscillation occurred, as shown in Fig 3 (a). In Fig. 3 (b), a solid line indicates the estimated friction force and a dashed line indicates the measured friction force obtained by Eq. (13). The average integral square error between the estimated and measured friction forces for the data set is 86.6. This value will hereafter be used as an evaluation criterion.

\subsection{Static friction component}

As shown in Fig. 3, the estimation update of this observer is inadequate at low velocities $(v \approx 0)$ because the pole of the observer corresponds approximately to zero. In order to avoid this phenomenon, Tafazoli et al. have suggested the modification of Eqs. (11) and (12) by considering the influence of a static friction force ${ }^{(11)}$. They use

$$
\hat{a}\left\{1-e^{-(v / \sigma)^{2}}\right\}
$$




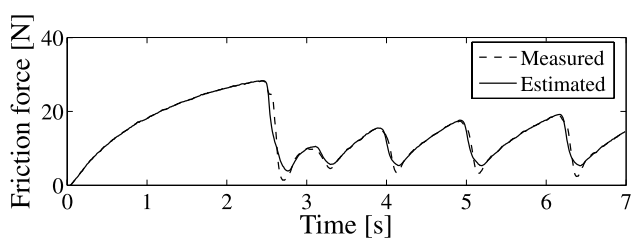

Fig. 5 Estimation of friction using the proposed observer

instead of $\hat{a}$ in Eq. (12), and moreover define the estimate of a friction force as

$$
\hat{f}+\left(f_{a}-\hat{f}\right) e^{-(v / \sigma)^{2}} .
$$

In this modification, inside the small neighborhood of $v=0$ defined by $\sigma$, static friction is dominant, and $\hat{f}$ is regarded as a function of $f_{a}$. Outside this neighborhood, the observer dynamics follow Eqs. (11) and (12).

In the same manner as described in the previous section, the data set was fed to this modified observer, and the average integral square error between the estimated and measured friction forces for it was calculated. A result of the experiments is shown in Fig. 4. The $\sigma$ value is decided by trial and error as $0.03 \mathrm{~m} / \mathrm{s}$ so as to minimize the estimation errors. A decrease of $17.6 \%$ in the average integral square error is obtained in comparison with the average error of the previous section. The modification provides a considerable improvement in the estimation performance, especially when operating at very low velocities, although the performance approaches that of the original observer at high velocities.

\subsection{Pressure-dependent component}

In addition to the above modification, we performed the further modification described below for the purpose of fitting the friction observer to a pneumatic cylinder. It is generally known that cylinder chamber pressure is exerted against the elastic seal, which is inserted between two sliding surfaces to prevent leakage, and that chamber pressure directly affects the friction force at the seal. As the pressure increases, the friction force increases as the seal is pressed against the inside surface of the cylinder chamber. For this reason, a friction force model with a pressure term has been developed ${ }^{(14),(15)}$. Also in our suggested observer, the pressure term, $M \alpha p_{m}$, is added to Eq. (11), and in accordance with this, Eq. (12) is replaced by

$$
\dot{z}=\left\{g_{a} \mu|v|^{\mu-1} \hat{a}-\alpha \dot{p}_{m}\right\} \operatorname{sgn}(v)
$$

where $\alpha$ is the design parameter used to adjust the contribution of the pressure term. The dynamics of the estimation error $\tilde{f}(=f-\hat{f})$ become

$$
\dot{\tilde{f}}=\dot{f}-g_{a} \mu|v|^{\mu-1} \tilde{f} .
$$

According to Eq. (17), it is guaranteed that the estimation error converges to zero if $g_{a}>0$, $\mu>0$, and $v \neq 0$ when assuming $\dot{f}=0$. Although this convergence condition is strict, it is the same as that of Eqs. (11) and (12). In addition to the incorporation of this pressure term, the modification represented by Eqs. (14) and (15) for expressing the influence of a static friction force is also introduced.

In order to verify the effectiveness of the modification proposed here, the estimation experiments were carried out for the same data set as that of $\S 3.1$. The design parameter $\alpha$ is determined as $1.5 \times 10^{-4} \mathrm{~m}^{2} / \mathrm{kg}$ through several estimation trials. Figure 5 shows a typical result of the tests. It was confirmed that the average integral square error between the estimated and measured friction forces for the data set decreases by $68.5 \%$ from that shown in $\S 3.1$ and that the estimation accuracy is much improved over that reported in the previous section. 


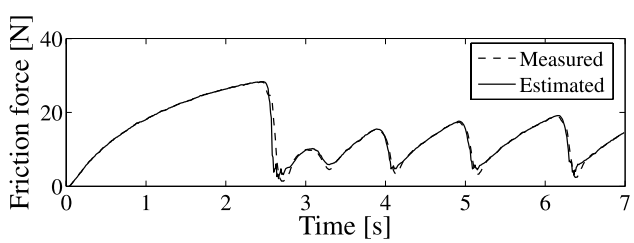

Fig. 6 Estimation of friction using the proposed observer with a velocity observer

Table 1 Values of system parameters

\begin{tabular}{|l|r|c|r|}
\hline$M$ & $5.33 \mathrm{~kg}$ & $x_{r}$ & $0.1 \mathrm{~m}$ \\
\hline$p_{0}$ & $0.2 \mathrm{MPa}$ & Sampling rate & $1 \mathrm{kHz}$ \\
\hline$g_{1}$ & $4.0 \times 10^{-3} \mathrm{~V} / \mathrm{Pa}$ & $g_{2}$ & $5.0 \times 10^{-7} \mathrm{Vs} / \mathrm{Pa}$ \\
\hline$g_{3}$ & $2.0 \times 10^{3} \mathrm{~N} / \mathrm{m}$ & $g_{4}$ & $1.0 \times 10^{2} \mathrm{Ns} / \mathrm{m}$ \\
\hline
\end{tabular}

\subsection{Simultaneous estimation of friction and velocity}

Our controller including the above friction observer requires the detection of piston velocity. Since eliminating the need for a velocity sensor can lead to cost reductions, and since a position sensor is available in our system, we use the velocity observer given by the following form $^{(10)}$ :

$$
\begin{aligned}
& \dot{z}_{v}=-g_{v} \hat{v} \\
& \hat{v}=z_{v}+g_{v} x
\end{aligned}
$$

where $\hat{v}$ is an estimate of the piston velocity, $z_{v}$ is the internal variable in the observer, and $g_{v}(>0)$ is the design parameter. The estimate $\hat{v}$ obtained by this observer is used in place of $v$ in both Eq. (9) and the friction observer. Figure 6 shows a result of the estimation tests using the above friction and velocity observers. The design parameter $g_{v}$ is selected as $g_{v}=$ $100 \mathrm{~Hz}$ such that the pole of the velocity observer is larger than that of the friction observer. Compared with the $\$ 3.1$ case, the estimation error decreases on average at $46.9 \%$. Although this estimation performance is higher than that shown in $\$ 3.2$, it is lower than that reported in the previous section. This difference occurs because the velocity observer composed of Eqs. (18) and (19) is a low-pass filtered differentiator and its estimation accuracy is not always high. However, this velocity observer does not require a model of a plant, is simple to design, and contributes to cost reductions.

\section{Experimental results}

Experiments were conducted to verify the performance of the proposed control on the pneumatic servo system shown in Fig. 1. The experiments used the parameter values listed in Table 1 . The values of the gains, $g_{1}-g_{4}$, were determined through rough tuning by simulations using Eqs. (1), (2), and (7) and fine tuning by experiments. Prior to the experiments, the following examination was carried out:

- The actual relation between the effective sectional area and the operating voltage of the valve is nonlinear, as determined by a series of measurements of the mass flow rate through the valve and the valve inlet and outlet pressures. Hence, the relation is linearized by applying its inverse relation ${ }^{(12)}$.

- The time constant of the inner pressure control system composed of Eqs. (5) and (6) became approximately $3 \mathrm{~ms}$ when we used the values in Table 1 . This result confirmed that the assumption that we made in $\$ 2.3$ is valid, even if the time lag in the valve action is added to the time constant.

For the performance evaluation, the following three cases were tested:

1 ) With Acceleration and Velocity Measurements: While implementing the control algorithm, the friction force that is calculated in real-time by substituting the accelerometer output and the pressure sensor outputs to Eq. (13) is sent to Eq. (9) ${ }^{(12)}$. The velocity sensor output is also sent to Eq. (9). 


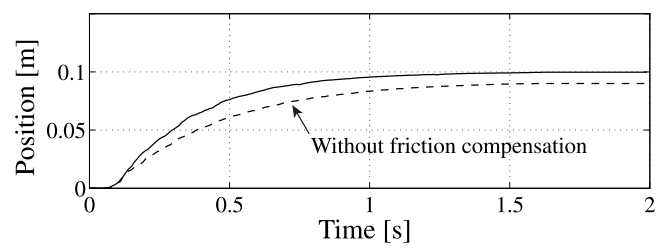

Fig. 7 Experimental response of positioning using the acceleration feedback

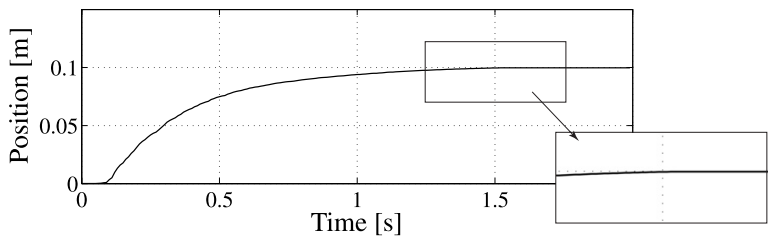

Fig. 8 Experimental response of positioning using the proposed friction observer

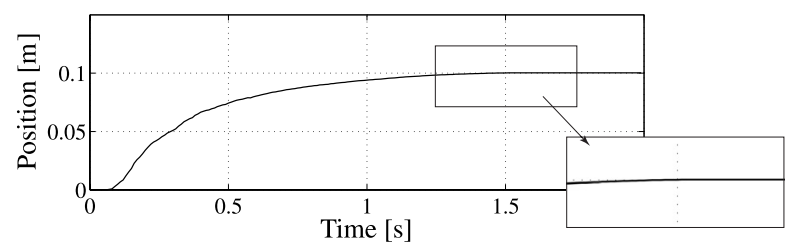

Fig. 9 Experimental response of positioning using the proposed observer with a velocity observer

2 ) With Friction Estimation and Velocity Measurement: The friction observer derived in $\S 3.3$ produces the friction estimate $\hat{f}$ based on the measured velocity and pressures. This $\hat{f}$ is used in Eq. (9) instead of $f$.

3 ) With Friction and Velocity Estimations: The friction observer is employed, but the velocity $v$ in this observer is replaced by the velocity estimate $\hat{v}$ as mentioned in $\$ 3.4$. This replacement is similarly made in Eq. (9).

Figures 7-9 illustrate representative results of the positioning experiments for a step input from the time $t=0$ for the cases 1 ) -3 ), respectively. A result of the experiments without the friction compensation (i.e. $F(s)=0$ in Eq. (9)) is also shown in Fig. 7 by a dotted line. Although slight adjustments of the gains, $g_{3}$ and $g_{4}$, from the values in Table 1 were needed for cases 2) and 3), almost the same responses were obtained as seen in these figures. The averages of the absolute values of the steady-state errors, which were calculated from ten trials each, are $0.086 \mathrm{~mm}$ for case 1), $0.075 \mathrm{~mm}$ for case 2), and $0.082 \mathrm{~mm}$ for case 3), and adequate tracking performances are realized. When the same positioning tests without the friction compensation were performed with all gains fixed and with all gains tuned, the steady-state errors became approximately $10 \mathrm{~mm}$ and $4 \mathrm{~mm}$, respectively. The initial dead time in the responses of Figs. 7-9 is caused by the time delay in valve spool motion, the time delay in pressure changes inside the cylinder chambers and pipes, and static friction.

Although the main part of our control algorithm consists of the widely used and simple PD control laws, and the auxiliary part of the algorithm is nothing but the computation procedure for the friction and velocity observers, the pneumatic servo system performed satisfactorily by implementing the algorithm. These results indicate that appropriately compensating for the friction is quite effective in improving the pneumatic system performance. In addition, compared to the system with an accelerometer and a velocity sensor, the proposed system with the friction and velocity observers instead of these sensors provided almost equivalent positioning accuracy despite its lower cost. Consequently, it was confirmed that the observerbased technique is favorable from the perspective of both cost and performance. 


\section{Conclusion}

In this paper, a positioning control system for a pneumatic actuator, whose performance is enhanced by introducing observer-based friction estimation and compensation, was developed. The results obtained in this study can be summarized as follows:

(1) By adding a model that describes the effects of pressure inside the chambers on the friction, a friction observer fitted to the pneumatic actuator was designed. Experimental investigations showed that this modification improved the estimation accuracy.

( 2 ) In our control system, the friction compensation was executed by regulating a desired pressure value used inside the system in accordance with the friction observer output. Using this compensation resulted in highly precise positioning in experiments, with a steady-state error of approximately $\pm 0.1 \mathrm{~mm}$.

( 3 ) The proposed system in which an accelerometer and a velocity sensor were replaced with a friction observer and a velocity observer in order to reduce costs exhibited almost the same performance as the system equipped with these sensing devices.

Currently, we are developing a pressure observer for a pneumatic actuator that acts as a software pressure transducer as a further cost-cutting measure in the positioning control system.

\section{References}

( 1 ) Noritsugu, T. and Takaiwa, M., Positioning Control of Pneumatic Servo System with Pressure Control Loop Using Disturbance Observer, Transactions of the Society of Instrument and Control Engineers, Vol. 31, No. 2 (1995), pp. 1970-1977.

( 2 ) Kadowaki, K., Inohara, K., Ishida, Y., and Notoyama, S., Robust Control for Pneumatic Cylinder Using $\mu$-synthesis, Journal of the Japan Fluid Power System Society, Vol. 29, No. 1 (1998), pp. 1-8.

( 3 ) Asakura, T., Takano, Y., and Guo, Q., Design of Robust Servo Control System for Pneumatic Manipulator, Transactions of the Japan Society of Mechanical Engineers, Series C, Vol. 64, No. 622 (1998), pp. 2124-2131.

( 4 ) Sakamoto, M., Yamada, Y., and Tanaka, M., Multi-Rate Neural Network type Model Reference Adaptive Control for Pneumatic Servo System, Transactions of the Japan Society of Mechanical Engineers, Series C, Vol. 67, No. 662 (2001), pp. 3153-3159.

( 5 ) Saito, T., Ikeda, H., Sugimoto, N., and Ikebe, J., A Study on the Low Velocity Control of a Pneumatic Cylinder, Journal of the Japan Hydraulics $\mathcal{E}$ Pneumatics Society, Vol. 26, No. 6 (1995), pp. 804-810.

( 6 ) Yang, Q.-H., Kawakami, Y., and Kawai, S., Position Control of a Pneumatic Cylinder with Friction Compensation, Journal of the Japan Hydraulics $\mathcal{F}$ Pneumatics Society, Vol. 28, No. 2 (1997), pp. 245-251.

( 7 ) Yoshimitu, T. and Oyama, O., Discrimination and Control of a Pneumatic Cylinder under Stick or Slide Mode of Seal, Journal of the Japan Fluid Power System Society, Vol. 33, No. 3 (2002), pp. 57-62.

( 8 ) Kosaki, T. and Sano, M., Gain-Scheduled $H_{\infty}$ Control of a Pneumatic Servo System for Compensating Nonlinear Friction Force, Transactions of the Japan Society of Mechanical Engineers, Series C, Vol. 67, No. 654 (2001), pp. 385-391.

( 9 ) Friedland, B. and Park, Y.-J., On Adaptive Friction Compensation, IEEE Transactions on Automatic Control, Vol.37, No.10 (1992), pp. 1609-1612.

(10) Amin, J., Friedland, B., and Harnoy, A., Implementation of a Friction Estimation and Compensation Technique, IEEE Control Systems Magazine, Vol. 17, No. 4 (1997), pp. 71-76.

(11) Tafazoli, S., W. de Silva, C., and Lawrence, P. D., Tracking Control of an Electrohydraulic Manipulator, IEEE Transactions on Control Systems Technology, Vol. 6, No. 3 (1998), pp. 401-411.

(12) Miyata, K. and Hanafusa, H., Velocity Control of Pneumatic Cylinders by Using Pres- 
sure Control, Transactions of the Society of Instrument and Control Engineers, Vol. 26, No. 7 (1990), pp. 773-779.

(13) Miyata, K., Ishida, K., and Hanafusa, H., Double Structured Feedback Control with Variable Gain Pressure Control System for Positioning of Pneumatic Cylinders, Transactions of the Society of Instrument and Control Engineers, Vol. 26, No. 7 (1990), pp. 787-794.

(14) Belforte, G., D'Alfio, N., and Raparelli, T., Experimental Analysis of Friction Forces in Pneumatic Cylinders, Journal of Fluid Control, Vol. 20, No. 1 (1989), pp. 42-60.

(15) Schroeder, L. E. and Singh, R., Experimental Study of Friction in a Pneumatic Actuator at Constant Velocity, Transactions of the ASME, Journal of Dynamic Systems, Measurement, and Control, Vol. 115 (1993), pp. 575-577. 